\title{
Glucose Tolerance versus HbA1c Results as Depictive of Gestational Diabetes Mellitus
}

\author{
Sikandar Hayat Khan1, Robina Manzoor2, Aasyia Hanif Baig2, Farah Sobia5, \\ Nadeem Fazal ${ }^{3}$ and Najmusaqib Khan Niazi ${ }^{4}$
}

\begin{abstract}
Objective: To evaluate glucose tolerance patterns in pregnant ladies undergoing 2-hour oral glucose tolerance test (OGTT) for comparing fasting, 1-hour, 2-hour post-glucose load results, HbA1c, sum of all glucose readings with and without gestational diabetes mellitus (GDM) using International Association of the Diabetes and Pregnancy Study Group (IADPSG) diagnostic criteria.

Study Design: Cross-sectional analysis.

Place and Duration of Study: PNS Hafeez, Naval Hospital, Islamabad, from January 2016 to July 2017.

Methodology: For 280 evaluated subjects reporting in mid-pregnancy for OGTT, results were segregated into four groups based upon comparison of 2-hour glucose result with 1-hour glucose. Group-1 2-hour results drop being $>2.0 \mathrm{mmol} / \mathrm{L}$ than1-hour results, group-2 with 2-hour result between $<2.0$ to $>0.5 \mathrm{mmol} / \mathrm{L}$ than peak at 1-hour, and group-3 with either 2-hour glucose drop being $<0.5 \mathrm{mmol} / \mathrm{L}$ or $>1$-hour results. Further, the ROC curve analysis was performed to compare the AUC for fasting plasma glucose, 1-hour post OGTT result, 2-hour post-OGTT result, factor additive of all OGTT readings and $\mathrm{HbA} 1 \mathrm{C}$.

Results: There was a progressive rise in $\mathrm{HbA1c}$ from group-1 to group-3 $(\mathrm{p}<0.001)$. Area under curve (AUC) for various diagnostic parameters for diagnosing GDM for additive value of all glucose results was 0.962 (95\% $\mathrm{Cl}: 0.935-0.988)$, 0.881 $(95 \% \mathrm{Cl}: 0.818-0944)$ for plasma glucose at 2-hour, for plasma glucose at 1-hour 0.898 (95\% Cl: $0.0 .842-0.954), 0.831$ $(95 \% \mathrm{Cl}: 0.0 .762-0.901)$ for fasting plasma glucose and $0.668(95 \% \mathrm{Cl}: 0.0 .578-0.759)$ for $\mathrm{HbA} 1 \mathrm{c}(\mathrm{p}<0.001)$.

Conclusion: Pregnant ladies demonstrating poor tolerance to glucose at 2-hour were observed to have higher $\mathrm{HbA1c}$ levels.
\end{abstract}

Key Words: Gestational diabetes mellitus, 2-hour OGTT, Glucose tolerance, International association of the diabetes and pregnancy study group (IADPSG) diagnostic criteria, Glycemic index, HbA1c.

\section{INTRODUCTION}

International Association of the Diabetes and Pregnancy Study Group (IADPSG) diagnostic criteria defines gestational diabetes mellitus (GDM) in pregnant ladies as hyperglycemia detected for the first time according to certain cut-offs of plasma glucose at fasting $(5.1 \mathrm{mmol} / \mathrm{L})$ and post-glucose load results at 1 -hour $(10.0 \mathrm{mmol} / \mathrm{L})$ and then 2-hour $(7.8 \mathrm{mmol} / \mathrm{L}) .^{1}$ These cut-offs, glucose loads, and number of testings have been varying since the inception of GDM screening. ${ }^{2-4}$

There are few issues with diagnostic testing of GDM after glucose loading, which needs to be appreciated while classifying subjects with GDM or non-GDM. Firstly, body mass index (BMI) can be a major factor while glucose loading among pregnant ladies as subjects with higher

1 Department of Pathology / Obstetrics \& Gynecology2 / Medicine 3 / Healthcare Administration 4 , PNS Hafeez, Naval Hospital, Islamabad, Pakistan

5 Department of Surgery, Combined Military Hospital (CMH), Multan, Pakistan

Correspondence: Dr. Sikandar Hayat Khan, Department of Pathology, PNS Hafeez, Naval Hospital, Islamabad, Pakistan E-mail:sik_cpsp@yahoo.com

Received: July 27, 2018; Accepted: November 23, 2018
BMI can have false negative result and vice versa. ${ }^{5}$ Weiss et al. suggested 100 or 75 gram glucose loading to be adjusted by a mathematical factor to have agreement between different strategies. ${ }^{6}$ Others have suggested different glucose loading criteria to reduce variability in results among pregnant ladies.7,8 Secondly, glucose absorption rates suffer from intra-subject bias among subjects as highlighted by Vogt et al. ${ }^{9}$ Chai et al. highlighted very high inter-individual biological variability for oral glucose tolerance test (OGTT) in comparison to $\mathrm{HbA1c}$ and fasting plasma glucose, due to differences in rates of glucose absorption resulting leading to show glucose peaks at different timings. ${ }^{10}$ Furthermore, 1 -hour testing requirement has already been obviated in nonpregnant subjects. ${ }^{11}$

Alongside, there is data to support that 2-hour glucose results are more associated with higher cardiovascular disease (CVD) risks. ${ }^{12}$ Asian population has been shown to be at more risk of both diabetes mellitus along with CVD in comparison to their Caucasian counterparts. ${ }^{13}$ The average height and BMI also differ among the European and sub-continental population, which is also associated with higher incidence of diabetes among the Asians. ${ }^{14}$ Molecular data pertaining to Asian population cohort have also suggested specific polymorphisms predisposing to the risk of GDM.15,16 Keeping the 
aforementioned controversies in GDM diagnosis, the authors aimed to evaluate glucose tolerance patterns in pregnant ladies undergoing 2-hour OGTT for comparing fasting, 1-hour, 2-hour post-glucose load results, HbA1c, sum of all glucose readings with and without GDM using IADPSG diagnostic criteria.

\section{METHODOLOGY}

This cross-sectional study was conducted at the Departments of Pathology and Gynecology, PNS Hafeez, Islamabad, with patient sampling started from January 2016 to July 2017 after formal approval of the project by the Hospital's Ethical Review Committee. Pregnant ladies in mid-pregnancy were requested to volunteer for participation into the study. After initial verbal approval and explanation of OGTT, study nature, protocols, probable side effects, and requirements of serial testing, they were requested to come as per American Diabetic Association (ADA) defined definition of fasting. Subjects who reported formally for study were enrolled at the Department of Pathology and were interviewed for related history and explanation of sampling, analytical process and data used for research purpose followed by a signed written consent on a structured format. Subjects who had known diabetes, hypertension, and related chronic or acute medical conditions or were taking drugs other than iron or folate supplements. Twenty more subjects, who could not complete tests, were also excluded due to their inability to tolerate glucose load, vomiting, sampling collection issues and could not turn up for the re-analysis.

OGTT was performed as per the IADPSG criteria. ${ }^{1}$ Fasting specimen was accompanied by more tests including glycated hemoglobin. Blood was collected in Na-Fluoride bottle for plasma glucose, plain bottle for serum ALT and EDTA tube for glycated hemoglobin and hemoglobin. We used Selctra-ProM for measuring glucose (GPO-PAP method) and ALT. HbA1c was measured by chemiluminescent microparticle immunoassay (CMIA) on ARCHITECT iSystem, Abbot diagnostics.

Subjects were divided out subjects based upon 2-hour results into three groups as: group-1 = post glucose load 2-hour glycemia $<2.0 \mathrm{mmol} / \mathrm{L}$ than peak at 1-hour; group-2 = post glucose load 2-hour glycemia $<1.5$ $\mathrm{mmol} / \mathrm{L}$ than peak at 1 -hour; and group-3 = post-glucose load 2-hour glycemia $<0.5 \mathrm{mmol} / \mathrm{L}$ than peak at 1 -hour or $>1$-hour results.
Data was initially entered into Excel programme and later moved to SPSS. Descriptive statistics for subjects were calculated using SPSS descriptive statistics. Comparisons among three groups based upon 2-hour post-glucose load test in comparison to 1-hour reading were compared using one-way ANOVA. Subjects having GDM or otherwise as per IADPSG criteria were compared first for the differences between each level of glucose, i.e., fasting, 1-hour, and 2-hour glucose readings by using independent sample test. Evaluating area under the curve (AUC) for all parameters including $\mathrm{HbA1c}$, fasting plasma glucose, 1-hour plasma glucose, 2-hour plasma glucose, and the additive value of all three glucose results was calculated through ROC analysis. The aforementioned OGTT based 3 results (fasting, 1-hour and 2-hour glucose results) based upon plasma glycemic patterns were evaluated for association with glycation status by one-way ANOVA.

\section{RESULTS}

Average age among our subjects were $28.26 \pm 4.83$ years with average BMI of $28.66(+4.82)$ with systolic blood pressure $103.41 \pm 6.90$ and diastolic blood pressure of $75.06 \pm 5.98 \mathrm{~mm}$ of $\mathrm{Hg}$. Mean parity status was $3.08 \pm 1.77$, with $1.36 \pm 1.25$ children, and abortions $0.17 \pm 0.65$. Table I shows the differences between fasting plasma glucose, 1-hour and 2-hour results among subjects with or without GDM at the given specified cut-

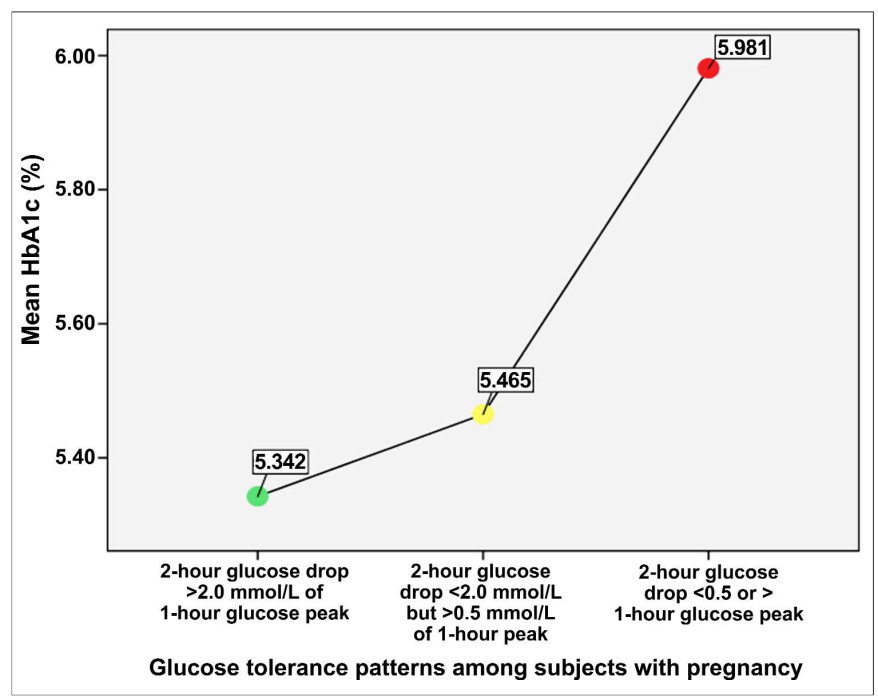

Figure 1: Differences in $\mathrm{HbA} 1 \mathrm{c}$ results based upon groups based upon glucose load tolerance by one way ANOVA $(n=280)$.

Table I: Differences between fasting plasma glucose, glucose result at 1- and 2-hour among subjects with or without GDM at specified cut-off at each level.

\begin{tabular}{|c|c|c|c|c|}
\hline Parameter & GDM diagnostic groups & $\mathrm{N}$ & Mean & Std. Dev \\
\hline \multirow[t]{2}{*}{ Fasting plasma glucose $(\mathrm{mmol} / \mathrm{L})$} & Gestational diabetes mellitus diagnosed & 50 & 5.37 & 1.59 \\
\hline & Gestational diabetes mellitus not diagnosed & 230 & 4.10 & 0.53 \\
\hline \multirow[t]{2}{*}{ Fasting plasma glucose at 1 -hour $(\mathrm{mmol} / \mathrm{L})$} & Gestational diabetes mellitus diagnosed & 50 & 10.67 & 3.22 \\
\hline & Gestational diabetes mellitus not diagnosed & 230 & 6.71 & 1.50 \\
\hline \multirow[t]{2}{*}{ Fasting plasma glucose at 2-hour (mmol/L) } & Gestational diabetes mellitus diagnosed & 50 & 8.88 & 3.41 \\
\hline & Gestational diabetes mellitus not diagnosed & 230 & 5.57 & 1.10 \\
\hline
\end{tabular}




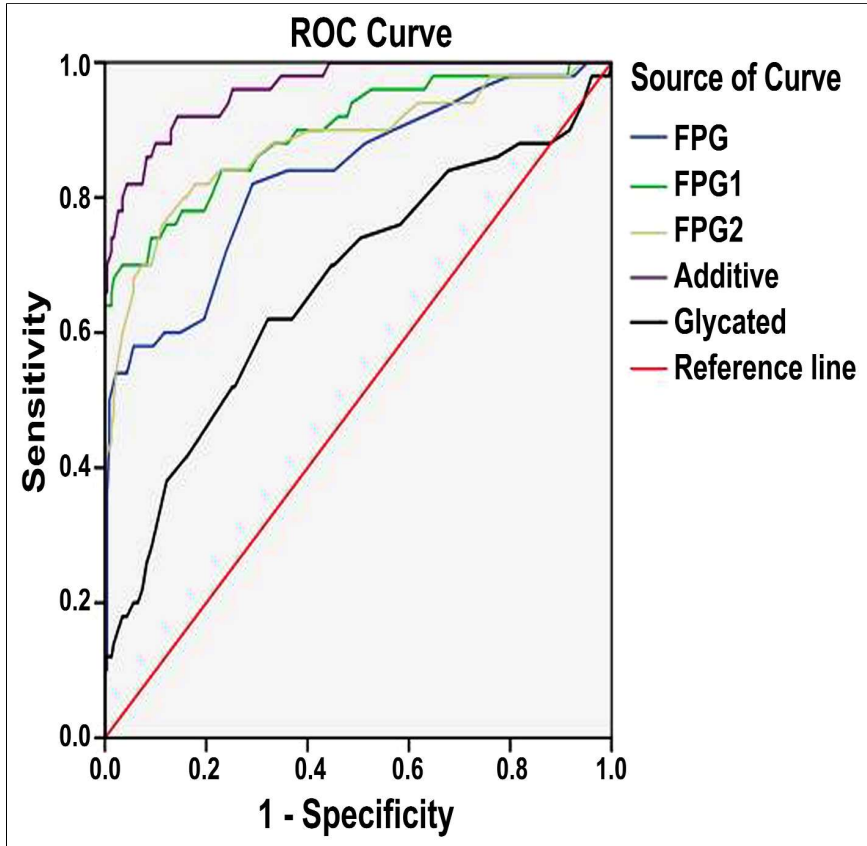

Figure 2: AUC for various diagnostic parameters for diagnosing gestational diabetes mellitus as: AUC for additive value of all glucose results $=0.962$ (95\% Cl: $0.935-0.988), A \cup C$ for plasma glucose results at 2 -hour $=0.881$ $(95 \% \mathrm{Cl}: 0.818-0944), \mathrm{AUC}$ for plasma glucose results at 1 -hour $=0.898$ (95\% Cl: $0.0 .842-0.954)$, AUC for fasting plasma glucose $=0.831(95 \% \mathrm{Cl}$ : $0.0 .762-0.901)$ AUC for $\mathrm{HbA} 1 \mathrm{c}=0.668$ (95\% Cl: 0.0.578-0.759), $(p<0.001)$.

offs. Figure 1 shows the results of three glucose readings during fasting, 1-hour and 2-hour during an OGTT, which indicated significant differences across all three readings. Figure 2 shows AUC for various diagnostic parameters for diagnosing gestational diabetes mellitus as being highest for additive value of all glucose results $=0.962$ (95\% Cl: 0.935-0.988), to lowest for HbA1c $=0.668(95 \%$ Cl: 0.0.578-0.759), $(p<0.001)$.

\section{DISCUSSION}

The study has highlighted two important aspects: Firstly, it was able to demonstrate that pregnant subjects who may not show a result crossing the given IADPSG cut-off still merit consideration due to possible low tolerance of that glucose load in that specific patient. So, a patient demonstrating slighter higher or showing a minimal drop by two hours in her plasma glucose, could have altered tendency towards glucose tolerance due to the stronger association between delayed glucose tolerance and raised glycation status as highlighted in our study. Though data may not be specific to GDM, it is available regarding delayed tolerance in GDM, still literature search yields indirectly provide evidence to support the present findings. 9,12,17 In the view of the authors, such a scenario can also happen in cases with GDM, with worst $\mathrm{HbA} 1 \mathrm{c}$ profiles in subjects having delayed rise or minimal drop in plasma glucose at 2-hour interval. Though literature review on the subject is quite variable with different screening modalities and cutoffs, 8,11 still 2-hour glucose result is more depictive of poor glucose tolerance as evident by some research.13,18,19
Finally, recent recommendations from NICE (UK) and $\mathrm{NIH}$ (USA) have not supported the IADPSG criteria and do not include 1-hour testing for GDM screening. 20-22

The second aspect, which our study has highlighted, is AUC for diagnosing subjects with GDM to be highest for combined sum of all the plasma glucose results during OGTT. While one glucose reading may or may not cross a borderline in random to compromise test efficiency, the combined utility of all values included within AUC cannot be undermined; and this concept may improve the diagnostic performance of OGTT. Corrado et al. have made use of AUC in suggesting screening for GDM.23 Similar evidence can also be found where area under the curve have been calculated to help diagnose GDM by incorporating multiple other factors. ${ }^{24}$

Certain limitations to the generalisability of this study results need to be acknowledged: Firstly, this crosssectional design was resource- and time-bound to follow these patients till pregnancy to associate maternal and fetal outcomes. Furthermore, like all cross-sectional studies this effort has been able to raise pertinent questions including utility of 1-hour result in OGTT, the concept of glucose tolerance rather than sampling climbing the cut-offs, and most significantly pertinent cut-offs for the Pakistani population.

Provided the limitations mentioned above, it is believed that the study is clinically important in providing regional pattern of glucose tolerance among pregnant subjects. The concept of given cut-offs to be more effective for the local population was also questioned, as slightly less glycemic tolerant subjects at 2-hour and subjects who showed delayed hyperglycemic peak, albeit not touching the IADPSG cut-offs were proven to have higher $\mathrm{HbA1c}$ levels, thus indicating a phenomenon which requires to be replicated through further well-controlled randomised trials in the Pakistani population.

\section{CONCLUSION}

Highest levels of HbA1c were in subjects who demonstrated a delayed 2-hour post-glucose load peak. The additive sum of all glucose readings showed the highest AUC for diagnosis of gestational diabetes followed by 1-hour and 2-hour glucose results.

\section{REFERENCES}

1. Metzger BE, Gabbe SG, Persson B, Buchanan TA, Catalano PA, Damm $\mathrm{P}$, et al. International association of diabetes and pregnancy study groups recommendations on the diagnosis and classification of hyperglycemia in pregnancy. Diabet Care 2010; 33:676-82.

2. Alberti KG, Zimmet PZ. Definition, diagnosis and classification of diabetes mellitus and its complications. Part 1: Diagnosis and classification of diabetes mellitus provisional report of a WHO consultation. Diabet Med 1998; 15:539-53.

3. [No authors listed]. Classification and diagnosis of diabetes mellitus and other categories of glucose intolerance. National Diabetes Data Group. Diabetes 1979; 28:1039-57. 
4. Carpenter MW, Coustan DR. Criteria for screening tests for gestational diabetes. Am J Obstet Gynecol 1982; 144:768-73.

5. Szczypaczewska M, Nazar K, Kaciuba-Uscilko H. Glucose tolerance and insulin response to glucose load in body builders. Int J Sports Med 1989; 10:34-7.

6. Gao X, Yan Y, Xiang S, Zeng G, Liu S, Sha T, et al. The mutual effect of pre-pregnancy body mass index, waist circumference and gestational weight gain on obesity-related adverse pregnancy outcomes: A birth cohort study. PLoS One 2017; 12:e0177418.

7. Weiss PA, Haeusler M, Kainer F, Pürstner P, Haas J. Toward universal criteria for gestational diabetes: Relationships between seventy-five and one hundred gram glucose loads and between capillary and venous glucose concentrations. Am J Obstet Gynecol 1998; 178:830-5.

8. Farrar D, Duley L, Dowswell T, Lawlor DA. Different strategies for diagnosing gestational diabetes to improve maternal and infant health. Cochrane Database Syst Rev 2017; 8:CD007122.

9. Vogt JA, Domzig C, Wabitsch M, Denzer C. Prehepatic secretion and disposal of insulin in obese adolescents as estimated by three-hour, eight-sample oral glucose tolerance tests. Am J Physiol Endocrinol Metab 2016; 311:E82-94.

10. Chai JH, Ma S, Heng D, Yoong J, Lim WY, Toh SA, et al. Impact of analytical and biological variations on classification of diabetes using fasting plasma glucose, oral glucose tolerance test and HbA1c. Sci Rep 2017; 7:13721.

11. Summary of technical report and recommendations. Report of a WHO/IDF Consultation. Available at: http://www.who.int/diabetes/ publications/Definition\%20and\%20diagnosis\%20of\%20diabet es_new.pdf (Retrieved on: 28-May-2018).

12. Hulman A, Simmons RK, Vistisen D, Tabák AG, Dekker JM, Alssema $\mathrm{M}$, et al. Heterogeneity in glucose response curves during an oral glucose tolerance test and associated cardiometabolic risk. Endocrine 2017; 55:427-34.

13. Lin YC, Chen HS. Longer time to peak glucose during the oral glucose tolerance test increases cardiovascular riskscore and diabetes prevalence. PLoS One 2017; 12:e0189047.

14. Ma RC, Chan JC. Type 2 diabetes in East Asians: Similarities and differences with populations in Europe and the United States. Ann N Y Acad Sci 2013; 1281:64-91.
15. Fatima SS, Chaudhry B, Khan TA, Farooq S. KCNQ1 rs2237895 polymorphism is associated with gestational diabetes in Pakistani women. Pak J Med Sci 2016; 32:1380-5.

16. Shi X, Cai Q2, Zou M, Shen Y. Correlation between TCF7L2 gene polymorphism and genetic susceptibility in women with gestational diabetes mellitus. Zhonghua Fu Chan Ke Za Zhi 2014; 49:588-93.

17. Ko GT, Cockram CS, Chan JC. How to minimize missing those subjects with high $2 \mathrm{HR}$ plasma glucose but 'normal' fasting plasma glucose levels? J Med 2001; 32:53-65.

18. Pimenta WP, Calderon IM, Cruz NS, Santos ML, Aragon FF, Padovani CR. Subclinical abnormalities of glucose metabolism in Brazilian women with a history of gestational diabetes mellitus. Acta Obstet Gynecol Scand 2004; 83:1152-8.

19. Lu W, Resnick HE, Jain AK, Adams-Campbell LL, Jablonski KA, Gottlieb AM, et al. Effects of isolated post-challenge hyperglycemia on mortality in American Indians: The strong heart study. Ann Epidemiol 2003; 13:182-8.

20. National Institute for Health and Care Excellence (NICE). Diabetes in pregnancy: Management of diabetes and its complications from preconception to the postnatal period. Clinical guideline NG3 (2015). Available at: https://www.nice.org.uk/ guidance/ng3/resources/diabetes-in-pregnancy-managementof-diabetes-and-itscomplications-from-preconception-to-thepostnatal-period-51038446021. Retrieved on: 29-May-2018.

21. Vandorsten JP, Dodson WC, Espeland MA, Grobman WA, Guise JM, Mercer BM, et al. NIH consensus development conference: diagnosing gestational diabetes mellitus. $\mathrm{NIH}$ Consens State Sci Statements 2013; 29:1-31.

22. Jacklin PB, Maresh MJ, Patterson CC, Stanley KP, Dornhorst A, Burman-Roy $S$, et al. A cost-effectiveness comparison of the NICE 2015 and WHO 2013 diagnostic criteria for women with gestational diabetes with and without risk factors. BMJ Open 2017; 7:e016621

23. Corrado F, D'Anna R, Cannata ML, Interdonato ML, Pintaudi B, Di Benedetto A. Correspondence between first-trimester fasting glycaemia, and oral glucose tolerance test in gestational diabetes diagnosis. Diabetes Metab 2012; 38:458-61.

24. Kondo M, Nagao Y, Mahbub MH, Tanabe T, Tanizawa Y. Factors predicting early postpartum glucose intolerance in Japanese women with gestational diabetes mellitus: Decision-curve analysis. Diabet Med 2018; 35:1111-7. 